\title{
Antimicrobial shortages: Another hurdle for clinicians
}

\author{
Louis Valiquette MD MSc FRCPC ${ }^{1}$, Kevin B Laupland MD MSc FRCPC ${ }^{2,3}$
}

$\mathrm{I}$ $\mathrm{n}$ an era of bacterial resistance, recently highlighted by the WHO global report on surveillance (1), choosing the best antimicrobial agent is more complex than ever. Prescribing the appropriate antimicrobial agent no longer involves simply selecting an empirical or definitive agent that will appropriately target the causative pathogens for a given patient; it also involves choosing the best agent for a given patient by taking into consideration the potential impact on bacterial resistance, lower risk for Clostridium difficile infection, lower toxicity, a schedule that improves compliance or discharge, etc (2).

The past several years have witnessed frequent shortages of antimicrobials and, more rarely, discontinuation of agents (3). These shortages pose substantial challenges, such as delays in initiation of the most optimal treatment, and increases in the use of agents with unnecessarily broad spectrums, as well as second- or third-line agents for which few clinical data are available; this can ultimately lead to suboptimal treatment and poorer outcomes (3). In addition, shortages may have a considerable impact on appropriate use in a manner that may be contrary to stewardship objectives.

Shortages occur either because of a decreased supply or an increased demand. The reasons for supply interruptions are typically not well known because manufacturers have no legal obligation of transparency. Manufacturing issues include raw material shortages, quality issues, capacity issues, manufacturing delays, etc. Increased demand can be associated with a new therapeutic usage, increase in incidence, stockpiling in preparation for announced outbreaks, etc $(4,5)$.

Antimicrobial shortages are not a new problem, but have clearly been increasing over the past two decades. According to the United States Food and Drug Administration (6), antimicrobial agents accounted for $13 \%$ of all drugs in shortage in 2011, second only to antineoplastic agents. From 2006 to 2010, the number of antimicrobial shortages increased significantly (3). In Canada, they are believed to be more prevalent in the generic drug market than in the brand name market.

Shortages vary among countries, suppliers and over time. In Canada, since 2012, the Canadian Drug Shortage Database, an industry-run website, provides information on current and resolved drug shortages. Although this tool facilitates access to important information, it is not perfect because shortage reporting by manufacturers in Canada was, until recently, performed on a voluntary basis. Also, compared with other resources, such as the American Society of Health-System Pharmacists Drug Shortages Resource Center (www. ashp.org/menu/DrugShortages.aspx), data are limited and no analyses of the potential impact on clinical care are provided. However, in February 2015, Canadian Health Minister Rona Ambrose announced new mandatory reporting regulations. Although this initiative alone will not end shortages, it could at least make updated and exhaustive information more readily available.
According to the Infectious Diseases Society of America, five important anti-infective injectables have a high probability of experiencing shortages in the near future: amikacin, clindamycin, trimethoprim/sulfamethoxazole (TMP/SMX), acyclovir and doxycycline (7). These shortages would have a significant impact on patient care and public health. However, none of the antimicrobial shortages that have recently impacted our clinical practice are even listed; these include cefepime, cefixime, ticarcilline-clavulanate and gentamicin. We will discuss their potential impacts and alternatives.

In September 2014, the sole Canadian manufacturer of adult preparations of gentamicin announced a shortage that would last several months. At the Centre Hospitalier Universitaire de Sherbrooke (CHUS, Sherbrooke, Quebec), to restrict the use of our limited stocks of gentamicin, we released local guidelines to automatically replace all gentamicin prescriptions with tobramycin unless patients fulfilled one of the criteria listed in Box 1. A switch to amikacin was recommended for treatment of a severe infection in which Serratia species are involved. As of March 2015, we are now facing a complete shortage of gentamicin; thus, we must seek alternatives to gentamicin for these patients.

In the specific situations listed in Box 1, other aminoglycosides cannot be used interchangeably. For enterococcal endocarditis, Enterococcus species are intrinsically resistant to tobramycin and amikacin. A priori, streptomycin may appear to be a potential alternative for enterococcal endocarditis because most laboratories can test strains for high-level resistance to streptomycin and its use is recommended in the American Heart Association endocarditis guidelines (8). However, increased use of streptomycin could rapidly end in a 'rebound' shortage because the demand and supply for this drug is usually limited, eg, for multidrug-resistant tuberculosis. Also, assessment of streptomycin serum concentrations requires special laboratory

BOX 1
Centre Hospitalier Universitaire de Sherbrooke
(Sherbrooke, Quebec) local guidelines for the use
of gentamicin
- As a synergistic agent in the treatment of all cases of presumed or
confirmed endocarditis due to Enterococcus species, Streptococcus
viridans or Streptococcus bovis with relative resistance to penicillin, or
for prosthetic endocarditis due to the same agents;
- As a synergistic agent in all suspected or confirmed cases of prosthetic
endocarditis due to Staphylococcus species;
- As a synergistic agent in all cases of culture-negative endocarditis or
suspected or confirmed Bartonella endocarditis;
- For the treatment of tularemia or brucellosis

${ }^{1}$ Department of Microbiology-Infectious Diseases, Université de Sherbrooke, Sherbrooke, Quebec; ${ }^{2}$ Department of Medicine, Royal Inland Hospital, Kamloops, British Columbia; ${ }^{3}$ Departments of Medicine, Critical Care Medicine, Pathology and Laboratory Medicine, and Community Health Sciences, University of Calgary, Calgary, Alberta

Correspondence: Dr Louis Valiquette, Department of Microbiology and Infectious Diseases, Centre hospitalier universitaire de Sherbrooke, 3001, 12ème Avenue Nord, Sherbrooke, Quebec J1H 5N4. Telephone 819-346-1110 ext 72584, fax 819-821-7101, e-mail louis.valiquette@usherbrooke.ca 
testing that is not readily available, making monitoring difficult. Finally, streptomycin is associated with a substantial risk for ototoxicity and vestibular disturbance. The same limitations would apply if streptomycin were used as an alternative to gentamicin for Bartonella endocarditis. For the latter infection, the American Heart Association suggests rifampin as an adjunct therapeutic agent to doxycycline if gentamicin is not available (8).

In vitro finding of amoxicillin and cefotaxime synergism against Enterococcus faecalis, based on differential saturation of penicillinbinding proteins, led researchers to perform observational studies to evaluate the potential of such a combination in E faecalis endocarditis (9). The most recent study, by Fernández-Hidalgo et al (10), demonstrated similar efficacy when comparing patients treated with ampicillin plus gentamicin versus ampicillin plus ceftriaxone using a nonrandomized, observational and nonblinded study design (10). Although a randomized blinded trial would be preferable, the gentamicin shortage may prompt clinicians to modify their practice before obtaining unequivocal data regarding this combination therapy.

Another antimicrobial agent that is no longer available in our practice is ticarcillin-clavulanate. For most indications, piperacillintazobactam, a similar, less expensive drug, is a suitable replacement. However, in patients infected with Stenotrophomonas maltophilia in whom TMP-SMX would be contraindicated (mainly because of allergies), ticarcillin-clavulanate is an interesting alternative. It is one of the only five antimicrobials, apart for TMP-SMX, for which the Clinical and Laboratory Standards Institute reports interpretive criteria (the others being ceftazidime, levofloxacin, minocycline and chloramphenicol) (11). Also, S maltophilia is not universally susceptible to these alternative drugs. According to the Canadian Antimicrobial Resistance Alliance, in 2013, only 27\% of the $67 \mathrm{~S}$ maltophilia isolates tested were susceptible to ceftazidime (12). From 494 (11\% of total) isolates collected in patients with pneumonia from $>50$ countries in Europe and United States as part of the SENTRY Antimicrobial Surveillance Program between 2009 and 2012, susceptibility to ceftazidime was $37 \%$, levofloxacin $75 \%$ and minocycline $99.5 \%$ (13). In vitro, minocycline and levofloxacin are appealing options; however, clinical data are limited to case reports and case series (14).

The Public Health Agency of Canada has reported a national cefixime shortage, which is expected to last until September 2015. In the past few decades, gonococcal infections have demonstrated increasing resistance to penicillin, tetracycline, azithromycin and quinolones (1). The proposed alternative is ceftriaxone $250 \mathrm{mg}$ intramuscularly (15). Restriction of administration of cefixime to patients for whom ceftriaxone use is not possible (mainly in settings where intamuscular administration would be difficult) has been proposed by the Agency. We recently treated a patient with clinical resistance to repeated azithromycin treatments and a history of anaphylactic allergy to penicillin. Fortunately, at the time of our consultation, an antibiogram was available and the strain was susceptible to ciprofloxacin. Otherwise, an admission to the intensive care unit for desensitization would have been the most likely option.

The final antibiotic for which we have experienced recent shortages is cefepime, a fourth-generation cephalosporin with extended spectrum of efficacy against Gram-negative (including Pseudomonas aeruginosa and AmpC beta-lactamase-producing Enterobacteriaceae) and Gram-positive bacteria. At the end of 2014, the brand manufacturer announced discontinuation of the product when their inventory is exhausted and the sole generic manufacturer is currently in shortage. There are several alternatives to cefepime in the management of patients with severe infections, and its shortage may appear to be of limited consequence. However, its unavailability could increase the selective pressure exerted by substitute drugs and may force prescribers to use agents with a broader spectrum of activity - two consequences that could contribute to the development of resistance.
Through these examples, we aim to illustrate that shortages are a real problem and that they impact clinical practice and patient care in Canada. Alternatives are not necessarily as optimal as the agents that are temporarily or permanently unavailable; they may be more toxic and lead to poor patient outcomes. Shortages can lead to increased costs because alternatives may be more expensive, length of stay may be prolonged because of slower clinical response and, finally, exceptional measures may be needed for infections classically treated in outpatient clinics. Forcing manufacturers to report anticipated shortages and discontinuations in Canada will improve clinicians' knowledge and preparedness, but will not tackle the problem at its root. Other strategies need to be identified and implemented at the federal level to promote an adequate supply of drugs, perhaps including incentives for manufacturers and wholesalers not to discontinue antimicrobial supply.

\section{REFERENCES}

1. World Health Organization. Antimicrobial resistance: Global report on surveillance. Geneva: WHO, 2014. < http://apps.who.int//iris/ handle/10665/112642> (Accessed March 19, 2015).

2. Dellit TH, Owens RC, McGowan JE Jr, et al. Infectious Diseases Society of America and the Society for Healthcare Epidemiology of America guidelines for developing an institutional program to enhance antimicrobial stewardship. Clin Infect Dis 2007;44:159-77.

3. Griffith MM, Gross AE, Sutton SH, et al. The impact of anti-infective drug shortages on hospitals in the United States: Trends and causes. Clin Infect Dis 2012;54:684-91.

4. ASHP Expert Panel on Drug Product Shortages; Fox ER, Birt A, James KB, Kokko H, Salverson S, Soflin DL. ASHP guidelines on managing drug product shortages in hospitals and health systems. Am J Health Syst Pharm 2009;66:1399-406.

5. Ventola CL. The drug shortage crisis in the United States: Causes, impact, and management strategies. P T 2011;36:740-57.

6. U.S. Food and Drug Administration. A Review of FDA's Approach to Medical Product Shortages. 2011 Contract No: March 15.

7. Relman DA. IDSA Comments on Drug Shortages Task Force and Strategic Plan 2013. <www.idsociety.org/uploadedFiles/IDSA/ Policy_and_Advocacy/Current_Topics_and_Issues/Advancing Product_Research_and_Development/Bad_Bugs_No_Drugs/ Position_Papers/IDSA Comments on Drug Shortages Task Force and Strategic Plan 031413.pdf> (Accessed March 19, 2015).

8. Baddour LM, Wilson WR, Bayer AS, et al. Infective endocarditis: Diagnosis, antimicrobial therapy, and management of complications: A statement for healthcare professionals from the Committee on Rheumatic Fever, Endocarditis, and Kawasaki Disease, Council on Cardiovascular Disease in the Young, and the Councils on Clinical Cardiology, Stroke, and Cardiovascular Surgery and Anesthesia, American Heart Association: Endorsed by the Infectious Diseases Society of America. Circulation 2005;111:e394-434.

9. Mainardi JL, Gutmann L, Acar JF, Goldstein FW. Synergistic effect of amoxicillin and cefotaxime against Enterococcus faecalis. Antimicrob Agents Chemother 1995;39:1984-7.

10. Fernández-Hidalgo N, Almirante B, Gavaldà J, et al. Ampicillin plus ceftriaxone is as effective as ampicillin plus gentamicin for treating Enterococcus faecalis infective endocarditis. Clin Infect Dis 2013;56:1261-8.

11. Clinical and Laboratory Standards Institute. Performance Standards for Antimicrobial Susceptibility Testing: Twenty-fifth Informational Supplement M100-S25. Wayne: Clinical and Laboratory Standards Institute, 2015.

12. Canadian Antimicrobial Resistance Alliance. 2015. <www.can-r. com/index.php $>$ (Accessed March 18, 2015).

13. Sader HS, Farrell DJ, Flamm RK, Jones RN. Antimicrobial susceptibility of Gram-negative organisms isolated from patients hospitalised with pneumonia in US and European hospitals: Results from the SENTRY Antimicrobial Surveillance Program, 2009-2012. Int J Antimicrob Agents 2014;43:328-34.

14. Falagas ME, Valkimadi PE, Huang YT, Matthaiou DK, Hsueh PR. Therapeutic options for Stenotrophomonas maltophilia infections beyond co-trimoxazole: A systematic review. J Antimicrob Chemother 2008;62:889-94.

15. Public Health Agency of Canada. Gonococcal infections. Canadian Guidelines on Sexually Transmitted Infections. Ottawa; 2013. 


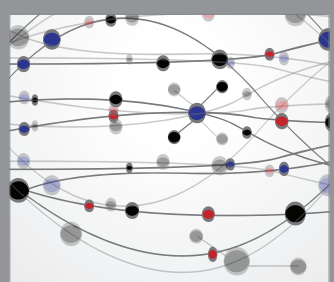

The Scientific World Journal
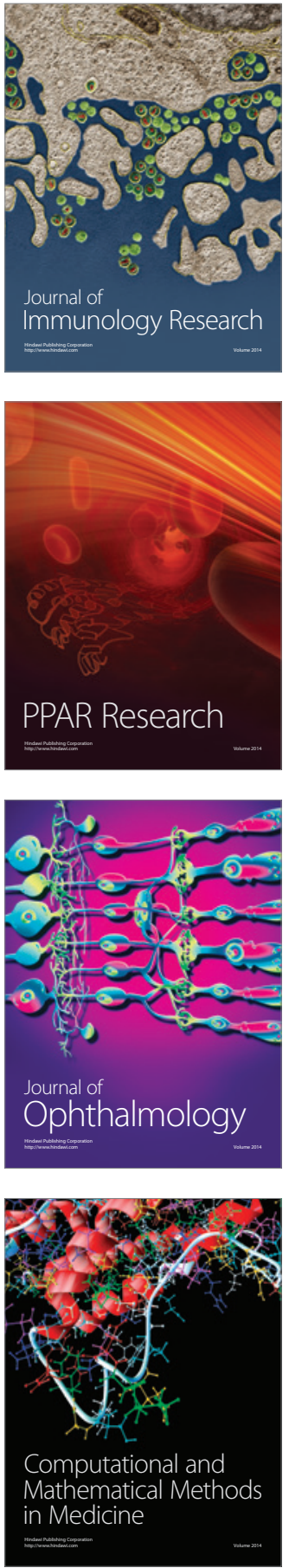

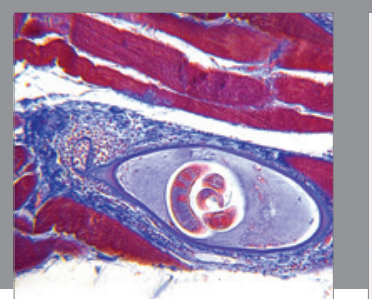

Gastroenterology Research and Practice

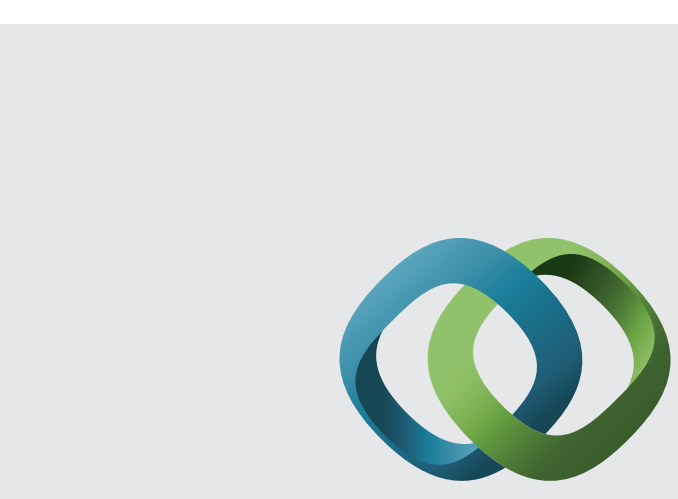

\section{Hindawi}

Submit your manuscripts at

http://www.hindawi.com
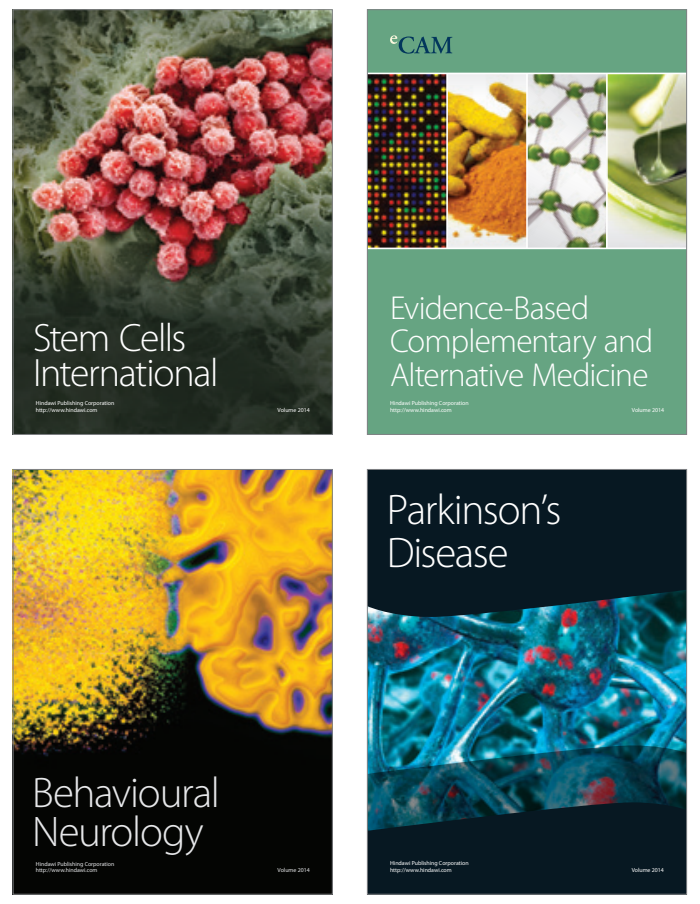
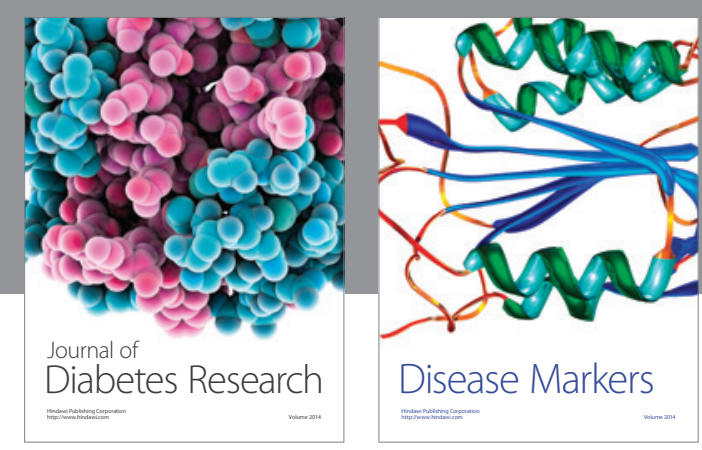

Disease Markers
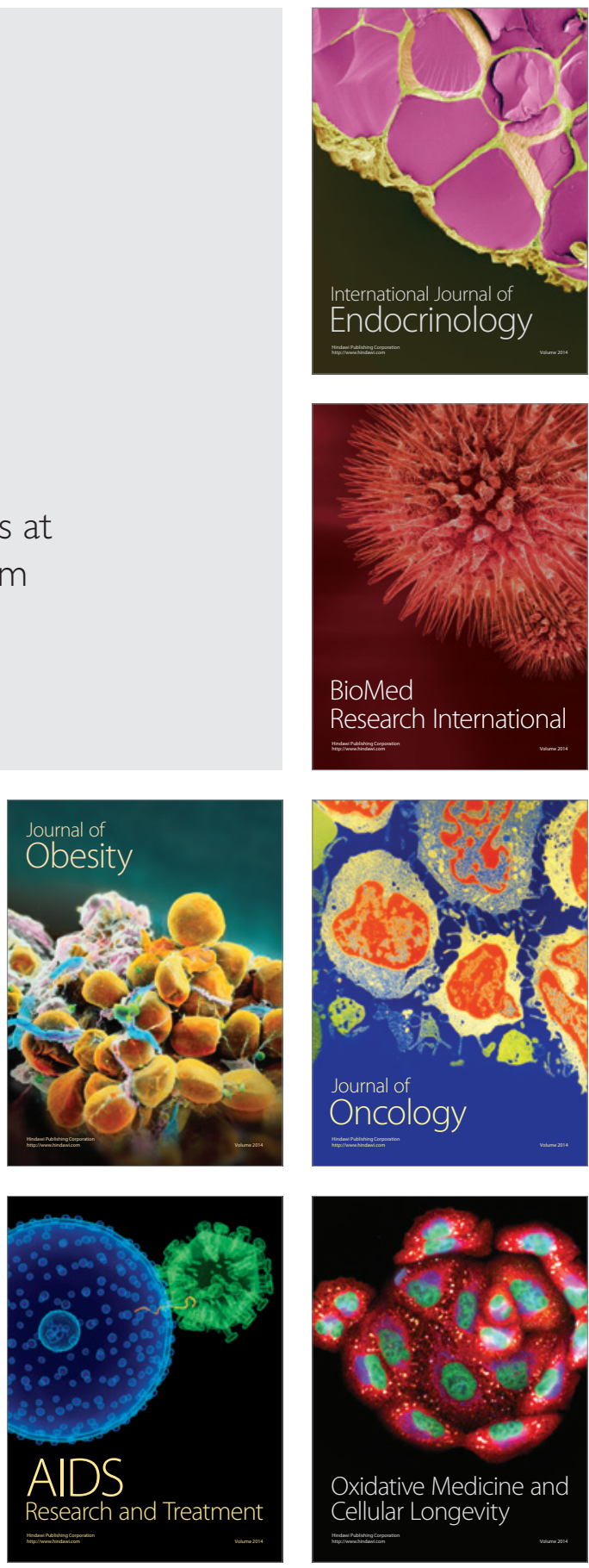